\title{
GRAU DE TRANSPARÊNCIA DE DADOS ABERTOS GOVERNAMENTAIS DO SITE DADOS.RS.GOV.BR
}

\section{DEGREE OF TRANSPARENCY OF GOVERNMENTAL OPEN DATA ON WWW.DADOS.RS.GOV.BR}

Rodrigo Hickmann Klein Pontifícia Universidade Católica do Rio Grande do Sul rodrigo.hickmann@acad.pucrs.br

Edimara Mezzomo Luciano Pontifícia Universidade Católica do Rio Grande do Sul eluciano@pucrs.br

Marie Anne Macadar Pontifícia Universidade Católica do Rio Grande do Sul marie.macadar@pucrs.br

Submissão: 06/04/2015

Aprovação: 20/10/2015 


\title{
RESUMO
}

Os Dados Abertos Governamentais tem o potencial de aumentar a eficiência na prestação de serviços e operações do setor público, bem como proporcionar a inovação em serviços governamentais em novos modelos de negócios e profissões. Além disso, proporcionam uma forma de apoiar e melhorar a interação entre o governo e os cidadãos, por incrementar a transparência governamental e fomentar a participação e colaboração. Este estudo de caso visa classificar o nível de transparência de um Portal de Dados no Brasil. Para atingir esse objetivo, adotou-se o modelo de mensuração de transparência dos Dados Abertos Governamentais de Veljković, Bogdanović-Dinić e Stoimenov (2014). Os resultados são relevantes para profissionais e pesquisadores interessados em avaliar a qualidade dos Portais de Dados Abertos Governamentais e desenvolver estratégias para promover um governo aberto.

Palavras-chave: transparência, dados abertos governamentais, governo aberto.

\begin{abstract}
Governmental open data have the potential to increase efficiency in public services as well as to provide innovation in government services with new business models and professions. They are also a way to support and improve the interaction between government and citizens by increasing government transparency and encouraging participation and collaboration. This case study aims to classify the level of transparency of a Brazilian open data portal. In order to achieve that, we adopted the Open Data measurement model presented by Veljković, Bogdanović-Dinic and Stoimenov (2014). The results are relevant to professionals and researchers interested in evaluating the quality of Open Government Data Portals and developing strategies to promote open government.
\end{abstract}

Keywords: transparency, governmental open data, open government. 


\section{Introdução}

No decurso das últimas décadas ocorreu uma mudança no enfoque do governo eletrônico, mudando gradativamente de ferramentas que incrementavam a conveniência da prestação de serviços do próprio governo para tornar-se também um facilitador da reforma administrativa e um promotor da participação democrática (YILDIZ, 2007). A título de exemplo, em setembro de 2011 foi lançada oficialmente uma iniciativa internacional, denominada Parceria para Governo Aberto (OGP na sigla em inglês), com o objetivo de difundir e incentivar globalmente práticas governamentais relacionadas à transparência dos governos, ao acesso à informação pública e à participação social. A parceria foi criada por oito países fundadores (África do Sul, Brasil, Estados Unidos, Filipinas, Indonésia, México, Noruega e Reino Unido) que assinaram a Declaração de Governo Aberto e apresentaram seus Planos de Ação. Atualmente, 60 países integram essa parceria, congregando nações e organizações da sociedade civil, pioneiros em transparência e governo aberto (OGP, 2014). O Brasil participa deste grupo desde 2011 como país-membro e assumiu o compromisso internacional de tornar efetiva a disposição dos dados públicos que possui.

Os Dados Abertos Governamentais (DAG), originados do Governo Aberto, contribuem para que ocorra um maior controle social e o fortalecimento da democracia e da cidadania ativa, bem como melhorias na administração pública, inovação, cooperação e transparência (HARRISSON et al., 2012). Os dados disponibilizados de forma gratuita a partir de organismos públicos podem ser usados para projetos da sociedade civil ou integrados em novos produtos, aplicativos ou serviços, tais como sistemas de navegação, previsões meteorológicas, ou serviços financeiros e de seguros. Como exemplo, podem ser utilizados como um dos critérios para cálculo da taxa de risco e da probabilidade de sinistro no trânsito, conforme os dados de ocorrências anteriores (DNIT, 2014).

No contexto apresentado, e considerando a importância crescente da transparência das ações dos governos, a questão de pesquisa é: como um portal de Dados Abertos Governamentais pode evoluir e se qualificar, em termos de transparência? O estudo de caso apresentado neste estudo apresenta uma alternativa de resposta a esta questão, classificando o grau de transparência de um portal de Dados abertos do Governo do Estado Rio Grande do Sul, utilizando o framework desenvolvido por Veljković, Bogdanović-Dinić e Stoimenov (2014), como prova de conceito em relação à mensuração de transparência dos dados. Este framework foi escolhido pela clareza e possibilidade de replicação e pela possibilidade de comparação com o portal data.gov do EUA, que foi um dos portais pioneiros da disponibilização de Dados Abertos Governamentais e referência aos demais (MCDERMOTT, 
2010).

Os resultados obtidos são relevantes para a prática dos gestores públicos que desejam avaliar a transparência dos portais de dados abertos e formular estratégias para divulgar os dados governamentais, bem como para pesquisadores deste tema.

\section{Referencial Teórico}

Para o alcance dos objetivos propostos neste estudo, é necessário a compreensão de dois conceitos fundamentais: transparência e dados abertos. Esta sessão busca discutir tais conceitos bem como trazer à tona terminologias que os tangenciam.

\section{Transparência}

O sentido de transparência adotado neste trabalho vincula o conceito ao relacionamento cidadão, em um sentido amplo de partes interessadas e que engloba pessoas físicas e jurídicas e o ente estatal. A transparência pode ser considerada como o esclarecimento prestado ao cidadão pelo Estado do que ocorre na sua esfera de competência, disponibilizando com presteza, rapidez e correção das informações que estão ao seu dispor. Assim, transparência significa deixar o Estado, no seu sentido mais amplo, aberto e visível ao cidadão (LHEUREUX-DE-FREITAS et al. 2013).

Por um lado, Dawes e Helbig (2010) vinculam a transparência à possibilidade do cidadão acompanhar realizações do governo e às medidas que este governo adota para vincular a responsabilidade dos representantes eleitos aos órgãos públicos, com relação às ações e decisões tomadas. As autoras também destacam que a transparência igualmente consiste em liberar dados e informações governamentais para que possam gerar valor econômico e social.

Por outro lado, Scholl (2012) relaciona o conceito de transparência ao compartilhamento da tomada de decisão, que em última análise para ele representa a participação. Segundo o autor, os governos, no futuro, atuarão sob intensos níveis de controle, avaliação e crítica públicos e os Dados Abertos são um dos mecanismos que permitirão tornar os governos mais transparentes. Assim, a transparência ajudará a responsabilizar os funcionários do governo por suas ações e omissões, e com o passar do tempo, a tomada de decisão governamental poderá se tornar amplamente compartilhada. Consequentemente, os governos que expõem seus dados de forma aberta, como por exemplo, através de Dados Abertos, poderão ser considerados governos abertos, desde que a transparência seja cotidiana e permanente (SCHOLL, 2012). 
Um outro importante aspecto levantado por De Ferranti et al. (2009), no que se refere à transparência, é a disponibilização pública e oportuna, com qualidade, abrangência e relevância, de informações confiáveis sobre as atividades do governo. Para os autores, estes são requisitos essenciais para se fornecer uma base contínua de aprovação do governante pelo cidadão. Abrange a divulgação voluntária e rotineira dos orçamentos, auditorias, políticas e ações executivas, tornando-se uma ferramenta para os cidadãos avaliarem a eficácia da ação administrativa e realizarem exigências sobre os serviços públicos que são prestados pelo governo. Esses atos, coincidentemente, geram pressão para melhorar o desempenho, fornecendo ao cidadão um feedback contínuo e permitindo avaliações mais abrangentes dos serviços governamentais (HARRISSON et al., 2012).

Nesse sentido Dawes (2010) detecta ênfase crescente, em nível mundial, no que se refere à transparência, mais especificamente ao acesso à informação, e que implica em duas tendências: [...] 1) direcionamento de mudanças no governo no sentido de ampliar a abertura e transparência; e 2) a disponibilidade e contínuo desenvolvimento de Tecnologias de Informação que permitem às instituições, organizações e indivíduos encontrar, compartilhar, combinar e reutilizar conteúdo de informação do governo (p.378).

\section{Breve evolução normativa brasileira}

Apesar de passos lentos, a legislação brasileira tem acompanhado a tendência mundial de adequar-se às mudanças exigidas pela sociedade no que se refere ao tema da transparência e acesso à informação. Em 1988, a carta magna já instituía o direito de acesso à informação pública no artigo $5^{\circ}$, inciso XXXIII do Capítulo I - dos Direitos e Deveres Individuais e Coletivos - o qual dispõe que: "todos têm direito a receber dos órgãos públicos informações de seu interesse particular, ou de interesse coletivo ou geral, que serão prestadas no prazo da lei, sob pena de responsabilidade, ressalvadas aquelas cujo sigilo seja imprescindível à segurança da sociedade e do Estado”. A Constituição Federal também tratou do acesso à informação pública nesse mesmo artigo, inciso XIV, e no artigo $37^{\circ}$, $\S 3^{\circ}$, inciso II (o da publicidade, que determina que atos públicos não podem ser sigilosos, salvo expressa disposição legal em contrário), bem como no Art. 216, § $2^{\circ}$.

Dois anos após a promulgação da Constituição Federal, a primeira regulamentação desta matéria é realizada por meio da Lei Complementar n. 101/2000 (Lei de Responsabilidade Fiscal - LRF), que estabelece normas de finanças públicas voltadas à 
responsabilidade na gestão fiscal e dá outras providências, sinalizou sobre a transparência na gestão fiscal. Posteriormente, a LRF foi alterada pela Lei Complementar $n^{\mathbf{0}} 131$, de 27 de maio de 2009. Esta lei assegura a disponibilização de informações relativas à gestão pública. Em seu artigo $1^{\circ}$, o diploma legal mencionado estabelece que a transparência deve ser assegurada como

I - inventivo à participação popular e realização de audiências públicas, durante os processos de discussão e elaboração de planos, leis de diretrizes orçamentárias e orçamentos;

II - liberação ao pleno conhecimento e acompanhamento da sociedade, me tempo real, de informações, de informações pormenorizadas sobre a execução orçamentária e financeira, em meios eletrônicos de acesso público;

Em seu artigo $2^{\circ}$, a norma determina a obrigatoriedade dos entes da federação, União, Distrito Federal, estados e municípios, permitirem o acesso a uma série de atos praticados pelas unidades gestoras relacionados às despesas e às receitas públicas. Foi a partir deste texto que a administração pública é obrigada a se reestruturar, de modo a poder construir e manter os "portais da transparência". Nestes sites os dados referentes à gestão orçamentária e financeira dos órgãos públicos (inclusive informações referentes a contratos e licitações) passam a ser obrigatoriamente disponibilizados. Este foi o primeiro passo para que o cidadão comum tivesse acesso a uma gama de consultas às informações da gestão pública que até então eram consideradas "caixa preta".

Logo em seguida ao surgimento desta lei, um novo marco legal, desta vez ampliando o espectro da transparência pública, emerge na realidade brasileira: a Lei no 12.5237 (BRASIL, 2011), sancionada pela Presidente da República em 18 de novembro de 2011, mais conhecida pela sigla LAI (lei de acesso à informação). Esta nova lei permite ao cidadão o acesso às informações cujo depositário é o Estado. A regra, a partir do ato legal, passa a ser a publicidade, e o sigilo, por consequência, se torna eventual e excepcional, condicionado à expressa previsão de lei. Com a entrada em vigor da LAI, em maio de 2012, o Brasil tornouse o $13^{\circ}$ país na América Latina e o $91^{\circ}$ país no mundo a conferir aos seus cidadãos mecanismos concretos para assegurar o direito de acesso à informação pública sob a guarda do Estado (MICHENER; MONCAU; VELASCO, 2014).

\section{Lei de Acesso à Informação (LAI)}

A LAI foi construída a partir de princípios universais norteadores do direito fundamental de acesso à informação (CONTROLADORIA-GERAL DA UNIÃO, 2013), quais sejam: 
- Publicidade máxima - respeitando preceitos internacionais e buscando uma mudança de paradigma em matéria de transparência pública, a LAI torna essencial o princípio de que o acesso deve ser a regra, e o sigilo, a exceção. Neste sentido, salvaguardandose os dados pessoais e as exceções expressas na lei, todas as demais informações são consideradas públicas e, por isso, passíveis de serem disponibilizadas aos cidadãos;

- Transparência ativa - não basta atender aos pedidos de informação, os órgãos públicos também devem publicar proativamente informações de interesse público;

- Abertura de dados - estímulo à disponibilização de dados em formato aberto, que podem ser livremente utilizados, reutilizados e distribuídos;

- Promoção de um governo aberto - sepultar a cultura do sigilo e estimular a cultura da transparência, uma vez que o acesso a informações é um direito humano fundamental;

- Criação de procedimentos que facilitem o acesso - agilidade, transparência, linguagem de fácil compreensão e estímulo ao uso de Tecnologia da Informação, sendo fundamental, também, a possibilidade de o cidadão recorrer da decisão em caso de negativa da informação.

O acesso é garantido de duas formas: ativa e passiva. A chamada transparência ativa ocorre quando o Estado concede proativamente, sem necessidade de pedidos, amplo acesso a informações de interesse coletivo. Já na transparência passiva, o Estado fornece informações específicas após sua solicitação por pessoas físicas ou jurídicas. Ao longo de seus artigos, a LAI deixa bastante evidente seu viés em prol da transparência ativa.

A LAI tem o propósito de regulamentar o direito constitucional de acesso dos cidadãos às informações públicas e seus dispositivos são aplicáveis aos três Poderes da União, Estados, Distrito Federal e Municípios. Estabelece que órgãos e entidades públicas devem divulgar informações de interesse coletivo, salvo aquelas cuja confidencialidade esteja prevista no texto legal. Isto deverá ser feito através de todos os meios disponíveis e obrigatoriamente em sites da internet. Entre as informações a serem disponibilizadas (transparência ativa) estão:

a) Endereços e telefones das unidades e horários de atendimento ao público;

b) Dados gerais para acompanhamento de programas, ações, projetos e obras;

c) Respostas a perguntas mais frequentes da sociedade;

Com o acesso prévio à informação (transparência ativa), o cidadão não precisa acionar o órgão, gerando benefícios para ele e economia de tempo e recursos para a administração.

Verifica-se que a LAI incentiva o uso de meios eletrônicos, obriga a divulgação de 
informações de interesse coletivo por meio da Internet e define critérios básicos de acessibilidade dos sites (ferramentas de pesquisa, gravação de dados, formatos de dados abertos, qualidade da informação, etc.). Quanto à transparência passiva, a LAI estipula procedimentos, normas e prazos para o processamento dos pedidos de informação e veda quaisquer exigências relativas aos motivos determinantes da solicitação.

\section{Dados Abertos}

Conforme a Organização para Cooperação e Desenvolvimento Econômico (OECD), a transparência é um princípio a ser considerado na construção de um Governo Aberto. No âmbito do Governo Aberto, a OECD identifica três principais princípios-chave: a) accountability: a necessidade de existência de mecanismos que possibilitem a identificação e responsabilização dos servidores públicos por suas ações; b) transparência: disponibilização de informações confiáveis, relevantes e tempestivas sobre as atividades do governo; c) participação social: o governo deve escutar os cidadãos e empresas, e considerar os seus anseios tanto no desenho quanto na implementação das políticas públicas (UBALDI, 2013). Dentre esses princípios-chave, a accountability se destaca, pois conforme Da Silva Júnior (2008), é um processo fundamental para a governança e governabilidade, pois envolve atores sociais nas diversas fases da gestão pública e implica assumir responsabilidades na condução do processo de análise e atendimento das demandas sociais.

\section{Princípios de Governo Aberto}

Entretanto, com relação ao Governo Aberto, a OGP — Parceria para Governo Aberto - (OGP, 2014) menciona cinco princípios que estão presentes em praticamente todas as definições sobre Governo Aberto e que, portanto, permitiriam classificar um governo como aberto: a) aumento da disponibilidade de informações sobre atividades governamentais; b) apoio à participação social; c) implementação de padrões mais altos de integridade profissional na Administração; d) aumento do acesso a novas tecnologias que promovam a transparência e accountability.

Dessa forma, como requisito essencial da transparência, a disponibilização dos Dados Abertos Governamentais representa a forma de tornar disponível a informação ao diversos segmentos interessados em seu conteúdo e garantir a transparência das informações do governo em longo prazo e, assim, contribuir para os direitos dos cidadãos ao acesso do público à informação do governo, que é considerado um dos princípios fundamentais da democracia (ZUIDERWIJK e JANSSEN, 2014). Além disso, políticas de dados abertos têm o potencial de aumentar a participação, a interação e a inclusão social dos usuários de dados 
abertos e fornecedores, e ao mesmo tempo, estimular o crescimento econômico (ONTSI, 2012).

Em 2007, na Califórnia (EUA), um grupo de trabalho designado para tratar da disponibilização das informações estatais desenvolveu os oito princípios sobre dados governamentais abertos, hoje universalmente aceitos pela comunidade mundial. Assim, dados governamentais são considerados abertos quando publicados de acordo com tais princípios a seguir definidos (DAVIES, 2013; UBALDI, 2013; OPEN GOVERNMENT DATA, 2015):

a) Completos: todos os dados públicos devem ser disponibilizados. Dado público é aquele que não está sujeito a restrições de privacidade, segurança ou outros privilégios;

b) Primários: o dado é apresentado tal como colhido da fonte, com o maior nível possível de granularidade, sem agregação ou modificação. Por exemplo, um gráfico não é fornecido aberto, mas os dados utilizados para construir a planilha que deu origem a ele podem ser abertos;

c) Atuais: o dado deve ser publicado o mais rápido possível, para preservar seu valor. Em geral, têm periodicidade, quanto mais recentes e atuais, mais úteis para seus usuários;

d) Acessíveis: os dados são disponibilizados para a maior quantidade possível de pessoas, atendendo, assim, aos mais diferentes propósitos;

e) Compreensíveis por máquina: devem estar estruturados de modo razoável, possibilitando que sejam processados automaticamente. Por exemplo, uma tabela em PDF é muito bem compreendida por pessoas, mas para um computador é apenas uma imagem, já uma tabela em formato estruturado, como CSV ou XML, é processada facilmente por softwares e sistemas;

f) Não discriminatórios: devem estar disponíveis para qualquer pessoa, sem necessidade de cadastro ou qualquer outro procedimento que impeça o acesso;

g) Não proprietários: Nenhuma entidade ou organização deve ter controle exclusivo sobre os dados disponibilizados;

h) Licenças livres: não devem estar submetidos a copyrights, patentes, marcas registradas ou regulações de segredo industrial.

Há restrições razoáveis quanto à privacidade, segurança e outros privilégios que são aceitas, desde que os argumentos sejam apresentados de forma transparente e bem justificados (UBALDI, 2013). 


\section{Benefícios na utilização de dados abertos governamentais}

Segundo a OECD (UBALDI, 2013), os Dados Abertos Governamentais fornecem uma plataforma para a prestação de serviços inovadores que podem resultar tanto da reutilização de dados por atores do setor privado quanto das organizações da sociedade civil. O uso de tecnologia de dados e análise dentro do setor público, bem como a integração da análise na formulação de políticas e concepção de serviço público, pode aumentar a entrega mais integrada e inovadora de serviços. No Reino Unido, por exemplo, as discussões entre o Departamento de Transportes e da Associação Comercial de empresas ferroviárias (ATOC) levou ao livre acesso aos bancos de dados de tarifas no início de 2013. Isto permitiu o desenvolvimento de sites de comparação de preços e aplicativos de telefonia móvel para o benefício de passageiros, que podem acessar informações precisas e encontrar passagens com os melhores preços.

A OECD destaca que um benefício potencial importante dos Dados Abertos Governamentais é a renda que pode ser gerada pela comercialização de dados do governo e o reuso dos DAG direta ou indireta em toda a economia (UBALDI, 2013). Isto pode levar a um aumento das receitas geradas a partir dos DAG, ou na criação de empregos em novos setores, como por exemplo, os infomediários, que intermediam os dados abertos governamentais realizando o cruzamento com demais dados, agregando valor, contexto e transformando-os em informação e conhecimento, e obtendo receitas diretas e indiretas com essa atividade (MAGALHAES et al., 2013). De acordo com uma pesquisa realizada pela Comissão Europeia em 2006, o tamanho do mercado global de informação do setor público na União Europeia (UE) está estimado, em média, em 27 bilhões de Euros, representando mais de 0,25\% do total do PIB agregado para a UE e Noruega (UBALDI, 2013). Uma análise econômica realizada por Vickery (2011) demonstrou que fornecer informações ao público gratuitamente, ou a um custo muito baixo, incentiva os cidadãos, desenvolvedores e empresas privadas a utilizar essas informações na criação de produtos para serem comercializados com maior valor agregado. Isso pode aumentar o volume de atividades do setor privado e estimular a economia nacional, como ocorreu na Espanha (ONTSI, 2012), e por consequência fornecer receita para o governo na forma de impostos (UBALDI, 2013), transformando a disponibilização dos dados em um investimento para o governo.

Os dados disponibilizados de forma gratuita a partir de organismos públicos podem ser usados para projetos da sociedade civil ou integrados em novos produtos, aplicativos ou serviços, tais como sistemas de navegação, previsões meteorológicas, ou serviços financeiros e de seguros, dentre outros. Como exemplo, podem ser utilizados como um dos critérios para 
cálculo da taxa de risco e da probabilidade de sinistros no trânsito, conforme os dados de ocorrências anteriores (DNIT, 2014), ou mensurar a influência dos gastos públicos sobre o PIB dos municípios (DE ANDRADE et al., 2014).

De acordo com um estudo finlandês (KOSKI, 2011), as empresas que reusam os dados geográficos cresceram 15\% a mais por ano em países onde os governos têm liberado essas informações livremente, em comparação com os países que tem cobrado por tais informações, a fim de recuperar os custos de produção.

$\mathrm{Na}$ Espanha, o projeto que disponibiliza os Dados Abertos Governamentais incentiva a reutilização de informações do setor público, fornecendo acesso a mais de 650 conjuntos de dados públicos em formatos reutilizáveis através de um portal de dados público, datos.gob.es. Em uma pesquisa realizada pelo Observatorio Nacional de las Telecomunicaciones y de la Sociedad de la Información da Espanha, foram identificadas um total de 150 empresas que atuavam exclusivamente no setor infomediário de informações de organizações públicas e privadas, empregando em torno de 4 mil pessoas e gerando em média 600 mil euros de faturamento. Nesta mesma pesquisa, os respondentes oriundos dessa atividade indicaram um potencial de faturamento anual neste setor na faixa de 330 a 550 milhões de Euros, que podem ser diretamente atribuídos à abertura dos dados governamentais e a sua reutilização, tornando-se um importante estímulo para o setor de TIC da Espanha (ONTSI, 2012).

\section{Processo de uso de dados aberto governamentais}

A utilização de Dados Abertos deve considerar alguns requisitos relativos ao licenciamento, pois por definição o Dado Aberto é aquele que pode ser livremente utilizado, reutilizado e redistribuído por qualquer pessoa, e deve, no mínimo, atribuir à fonte original ou fazer o compartilhamento desses dados utilizando a mesma licença em que as informações foram apresentadas. A definição completa de dados abertos fornece detalhes específicos do significado do termo, que se resumem em: a) disponibilidade de acesso: o dado precisa estar disponível por inteiro e por um custo razoável de reprodução, preferencialmente, sem custo, por meio de download na Internet, e também deve estar em um formato conveniente e modificável; b) reuso e redistribuição: o dado precisa ser fornecido em condições que permitam a sua reutilização e redistribuição, incluindo o cruzamento com outros conjuntos de dados; c) participação universal: todos podem usar, reutilizar e redistribuir, não havendo discriminação contra áreas de atuação, pessoas ou grupos (DAVIES, 2013).

Segundo a OECD, os Dados Abertos Governamentais tem o potencial de aumentar a eficiência do governo e a inovação na prestação de serviços e operações internas do setor 
público (UBALDI, 2013), pois a publicação de dados contribui para a mitigação significativa das solicitações de informações rotineiramente ao governo, possibilitando que as solicitações sejam acessadas diretamente pela população e trocada entre os entes governamentais. Os benefícios subsequentes incluem a carga de trabalho reduzida, a redução da burocracia e a redução dos custos de transação. Conforme a $\mathrm{OECD}$, quatro fases principais podem ser identificadas nessa cadeia de valor dos Dados Abertos Governamentais (UBALDI, 2013):

a) Geração de dados: abrange a geração de dados públicos, o que normalmente é feito por entidades do setor público, mesmo que esta função possa se tornar cada vez mais compartilhada com outras fontes de dados com financiamento público (por exemplo, estatísticas sociais, dados aéreos);

b) Coleta de dados, agregação e processamento: os dados precisam ser coletados, agrupados para permitir o acesso, compartilhamento e reutilização, a fim de agregar valor para a maioria dos usuários, como por exemplo, no apoio a decisões;

c) Distribuição de dados e entrega: os dados precisam ser distribuídos para os potenciais usuários e para permitir o acesso e reutilização;

d) Utilização dos dados finais: os dados do governo aberto precisam ser reutilizados por toda uma relação diferentes de usuários para sustentar a criação de valor público.

Todavia, conhecer a cadeia de valor DAG é essencial para compreender o papel atual dos principais atores e entender como a velocidade de evolução desse novo mercado está causando mudanças significativas nos papéis e limites das respectivas responsabilidades. Nesse sentido, a Deloitte (DELOITTE ANALYTICS, 2013) realizou a análise de uma série de empresas desse novo segmento de mercado e identificou modelos de negócios diferentes de utilização dos DAG na geração de receitas, que podem ser agrupados em torno de cinco arquétipos:

a) Fornecedores: que publicam dados, incluindo o setor público;

b) Agregadores: combinam os dados publicamente disponíveis com novos dados para produzir informações úteis a vários usuários diferentes;

c) Desenvolvedores de aplicativos: desenvolvem aplicativos para a tomada de decisão, como por exemplo, aplicativos com base nos dados de criminalidade, dados de transporte;

d) Enrichers: empresas que produzem quantidades significativas de dados abertos e combinam os Dados Abertos Governamentais com suas próprias fontes proprietárias de dados para realizar prestação de serviços com esses dados 
combinados, como por exemplo, a seguradoras e varejistas;

e) Facilitadores: organizações que não obtém recursos a partir dos dados abertos, mas fornecem plataformas e tecnologias que podem ser utilizadas pela sociedade, como por exemplo, sites que permitem combinar várias fontes e tipos de dados, criando novos subconjuntos de dados.

Perante esse contexto, pode-se considerar a participação como uma ferramenta para o aprimoramento das decisões políticas e serviços públicos, incluindo a colaboração dos cidadãos, empresas e agências governamentais, em tarefas ou projetos que visam produzir resultados específicos (LEE e KWAK, 2011).

\section{Método de Pesquisa}

A presente pesquisa adotou uma abordagem exploratória, com a utilização de dados secundários coletados via pesquisa documental. Embora tal estudo tenha abordado um site em específico, as estratégias praticadas por diversos governos em suas esferas e poderes são diferenciadas, razão pela qual foi utilizada a abordagem exploratória. Utilizando como base o referencial teórico exposto, buscou-se um modelo que pudesse mensurar o grau de transparência de dados em um contexto regional. Dessa forma, optou-se por mensurar o grau de transparência dos dados do catálogo de fontes de dados disponibilizados no site http://dados.rs.gov.br. Os dados foram tratados com técnicas estatísticas definidas no framework de Veljković, Bogdanović-Dinić e Stoimenov (2014), com aproveitamento do material textual que foi coletado a partir do acesso ao site dados.rs.gov.br, nos meses de junho a agosto de 2014.

Optou-se por este modelo por ser abrangente, atual e com maior clareza de metodologia. Neste modelo os dados abertos são usados como uma fonte de dados para três indicadores: conjunto de dados, abertura de dados e transparência de dados. O envolvimento do usuário é utilizado como fonte de indicadores de participação e colaboração. Como o foco deste artigo são os dados abertos, os indicadores são fundamentados, incluindo o conjunto de dados, abertura dos dados e transparência dos dados, que serão descritos no decorrer deste artigo.

Para estabelecer um modelo de avaliação padrão é crucial um conjunto básico de categorias a serem definidos e adotados. Com base em pesquisas em portais de dados abertos em vários países, Veljković, Bogdanović-Dinić e Stoimenov (2014) destacam nove categorias de dados mais comumente disponíveis: finanças e economia, meio-ambiente, saúde, energia, 
educação, transporte, infraestrutura, emprego e população. Consequentemente, estas categorias foram impostas para formar o conjunto de dados básicos pré-definido. Uma categoria é considerada como existente caso possua, ao menos, um conjunto de dados publicados. Conforme os autores do modelo, os portais dos governos devem ter todas as nove categorias de dados a partir de um conjunto de dados básicos (dataset), juntamente com os dados correspondentes para cada categoria.

O indicador básico do conjunto de dados (BSD) determina a presença de um conjunto predefinido de alto valor dentre as categorias de dados aberto e recebe valores entre 0 (zero) e 1 (um). O cálculo é realizado de acordo com a seguinte equação:

$$
B S D=n / N \text {. }
$$

O valor "n" representa o número de categorias que estão presentes no portal de dados abertos, enquanto que o $\mathrm{N}$ marca o número total de categorias $(\mathrm{N}=9)$. $\mathrm{O}$ valor 0 (zero) indica que não existe nenhuma categoria ou as categorias ainda não possuem um conjunto de dados disponibilizados no portal, enquanto que o valor 1(um) indica que todas as categorias estão presentes.

Em virtude das características da pesquisa, em especial pela utilização do framework de benchmarking de Veljković, Bogdanović-Dinić e Stoimenov (2014), estabeleceu-se um processo dinâmico de coleta-análise de dados: cada conjunto de dados era localizado (ou não) no portal, as características destes conjuntos de dados eram identificadas e organizadas em planilhas e em seguida as equações do framework eram aplicadas. Desta forma, pode-se dizer que ocorreu uma análise de conteúdo utilizando as categorias definidas previamente pelo framework utilizado como base para esta pesquisa, tendo como resultado desta análise a classificação e entendimento detalhado dos conjuntos de dados.

O Apêndice explica detalhadamente os procedimentos de análise de dados e mostra os resultados das análises, sendo assim um importante parte do texto para entender como as análises foram operacionalizadas.

\section{Resultados}

No caso do portal dados.rs.gov.br, somente as categorias demonstradas na Tabela 1 possuíam conjuntos de dados disponibilizados.

Tabela 1 - Categorias e tamanho de amostra

\begin{tabular}{|l|c|}
\hline Categorias/Subcategorias & Conjunto de dados \\
\hline Finanças & 8 \\
\hline Finanças/Desenvolvimento & 4 \\
\hline Saúde & 5 \\
\hline
\end{tabular}




\begin{tabular}{|l|c|}
\hline Educação & 17 \\
\hline Transporte (Trânsito) & 7 \\
\hline População & 4 \\
\hline Total & $\mathbf{4 5}$ \\
\hline
\end{tabular}

Em relação à quantidade de categorias presentes no portal dados.rs.gov.br, o BSD obtido foi 0,56 , que é considerado ainda mediano em comparações à pesquisa de Veljković, Bogdanović-Dinić e Stoimenov (2014), por exemplo o portal data.gov do EUA atingiu 0,89 de BSD. O resultado é devido à falta das categorias Meio Ambiente, Energia, Infraestrutura e Emprego. Entretanto, diversas outras categorias não indicadas pelos autores fazem parte do portal dados.rs.gov.br, como por exemplo, Segurança e Representação Política, perfazendo entre todas as categorias disponíveis 70 conjuntos de dados.

Outro indicador utilizado é o Indicador de Abertura de Dados (DO), que visa avaliar o percentual de abertura dos dados publicados e é composto por oito critérios que são consistentes com os oito princípios para os Dados Abertos oriundos do Grupo de Trabalho do Governo Aberto (DAVIES, 2013).

O Quadro 1 fornece uma breve descrição de cada um dos critérios, juntamente com os seus valores e um valor total para o indicador de abertura de dados.

Quadro 1 - Critérios para cálculo de abertura dos dados (DO)

\begin{tabular}{|c|c|c|c|c|}
\hline \multicolumn{4}{|c|}{ Critérios com base nos princípios dos Dados Abertos Governamentais } & \multirow{2}{*}{$\begin{array}{c}\begin{array}{c}\text { Máximo de } \\
\text { pontuação por } \\
\text { critério }\end{array} \\
1 \text { ponto }\end{array}$} \\
\hline Completo & \multicolumn{3}{|c|}{ Os dados estão disponibilizados integralmente? } & \\
\hline Primário & \multicolumn{3}{|c|}{ O dados são fornecidos em formato original e podem ser utilizados para análises futuras? } & 1 ponto \\
\hline Atuais & $\begin{array}{l}\text { O intervalo de tempo } \\
\text { relatado pelo dados é } \\
\text { mencionado: } \\
0,3 \text { ponto }\end{array}$ & $\begin{array}{l}\text { A frequência de atualização dos } \\
\text { dados é mencionada: } \\
0,4 \text { ponto }\end{array}$ & $\begin{array}{l}\text { A última atualização, conforme a } \\
\text { frequência e intervalo está } \\
\text { disponibilizada: } \\
0,3 \text { ponto }\end{array}$ & 1 ponto \\
\hline Acessiveis & \multicolumn{3}{|c|}{ Os dados estão disponiveis para qualquer pessoa, para qualquer propósito? } & 1 ponto \\
\hline $\begin{array}{l}\text { Compreensiveis por } \\
\text { máquina: }\end{array}$ & $\begin{array}{l}\text { Formato .PDF ou } \\
. \text { XLS } \\
0,2 \text { ponto }\end{array}$ & $\begin{array}{l}\text { Formato CSV: } \\
0,5 \text { ponto }\end{array}$ & $\begin{array}{l}\text { Formato XML ou RDF (Resource } \\
\text { Description Framework): } \\
1 \text { ponto }\end{array}$ & 1 ponto \\
\hline Não discriminatórios & \multicolumn{3}{|c|}{ Os dados são de livre acesso? } & 1 ponto \\
\hline Não proprietários & \multicolumn{3}{|c|}{ Os dados estão disponiveis em formatos não proprietários } & 1 ponto \\
\hline Licenças Livres & \multicolumn{3}{|c|}{ Os dados estão publicados sobre licenças abertas? } & 1 ponto \\
\hline
\end{tabular}

Fonte: Veljković, Bogdanović-Dinić e Stoimenov (2014)

A integralidade dos dados é calculada de acordo com cinco características 
reconhecidas: a presença de uma descrição dos metadados, a possibilidade de transferência de dados, se os dados são legíveis por máquina e se os dados possuem hiperlink para facilitar a acessibilidade, como, por exemplo, disponibilizar um hiperlink para os dados em uma aplicação web. Os dados são considerados primários se são publicados em formato original, para permitirem diferentes análises. Caso os dados sejam publicados sob a forma de um gráfico, ou qualquer outro formato pré-analisado, os dados não são considerados como primários. Cada conjunto de dados deve ter em sua descrição o período de tempo abrangido pelos dados, a frequência de atualização e quando foi realizada a última atualização.

A acessibilidade (A) de dados considera que os dados devem ser acessíveis a todos igualmente, sem solicitar o motivo do acesso ao usuário, e não discriminatório, sendo disponíveis gratuitamente. Não obstante, devem ser processáveis por máquina, ou seja, os dados devem ser processáveis por um computador. Sendo assim, o modelo proposto reconhece três níveis de avaliação: a) formatos que não são processáveis por máquina, como exemplo, PDF; b) formatos estruturados que podem ser processados automaticamente, por exemplo, CSV; c) formatos, que incluem meta descrição e semântica, por exemplo, XML e RDF (Resource Description Framework).

Os recursos não proprietários se relacionam com o critério anterior, pois considera o licenciamento dos formatos dos dados: para conjuntos de dados que estão disponíveis em um formato como XLS é atribuído o valor 0 (zero), enquanto para formatos livres e abertos como CSV, XML ou RDF é atribuído o valor 1. Por fim, a característica de licença livre é pontuada com 1 se o conteúdo dos dados é publicado sob uma licença aberta.

A abertura de dados para todo o portal foi calculada como um valor médio de abertura de todas as categorias de conjuntos de dados. A Tabela 2 apresenta uma escala de abertura de cinco níveis que se baseia em valores calculados para o indicador DO.

Tabela 2 - Níveis de Abertura dos Dados

\begin{tabular}{|l|l|}
\hline Indicador DO & Nível de Abertura dos Dados \\
\hline $0 \%-5 \%$ & Inicial \\
\hline $6 \%-35 \%$ & Abertura básica \\
\hline $36 \%-75 \%$ & Abertura mediana \\
\hline $76-90 \%$ & Abertura \\
\hline$>90 \%$ & Altamente aberto \\
\hline
\end{tabular}

Fonte: Veljković, Bogdanović-Dinić e Stoimenov (2014)

O indicador de dados para toda a abertura do portal pode ter valores que estão no 
intervalo $0(0 \%)$ e 1 (100\%). O nível mais baixo é chamado de inicial, e ele só reconhece uma iniciativa para a adoção de abertura de dados, enquanto outros níveis identificados indicam avanço do governo em relação a oito princípios de abertura.

Os resultados detalhados da análise Indicador de Abertura de Dados (DO) do portal dados.rs.gov.br constam no Apêndice. Este Indicador obteve o percentual de 36,47\%, abaixo da média obtida por Veljković, Bogdanović-Dinić e Stoimenov (2014) com o portal dados.gov que foi $67,5 \%$. Esse valor inferior se deve, principalmente, à falta de informações sobre licenciamento do conteúdo dos dados disponibilizados no portal dados.rs.gov.br, que prejudicam a sua reutilização, pois não está claro o nível de detalhamento do referenciamento necessário à fonte dos dados, ou se os dados poderão ser utilizados livremente sem ônus ao usuário. Outros fatores que influenciaram no baixo DO foi o grau de atualização dos dados, e por último os formatos, que em alguns casos disponibilizados eram apenas disponibilizados em formato PDF.

Para calcular o grau de transparência do portal dados.rs.gov.br foi utilizado o indicador de transparência $(\mathrm{T})$, composto por outros dois indicadores, Transparência do Governo (GT) e Dados de Transparência (DT), e é calculado pela média dos seus valores. Conforme apresentado abaixo, será utilizada a média desses dois valores. Veljković, Bogdanović-Dinić e Stoimenov (2014) consideram que os dois tipos de transparência, de dados e do governo, são igualmente importantes para a construção de um governo transparente. $\mathrm{O}$ indicador $\mathrm{T}$ tem seus valores a partir do intervalo $(0,1)$, onde 0 (zero) significa a falta de transparência e 1 (um) está associada à alta transparência.

$$
\mathrm{T}=\mathrm{GT}+\mathrm{DT} / 2
$$

A Transparência do Governo é observada como uma medida de percepção de tarefas do governo, processos e operações. O indicador de Transparência dos Dados (DT) é calculado como uma média dos valores de autenticidade (A), Compreensibilidade (U) e reutilização dos dados, conforme apresentado abaixo. Este indicador tem valores a partir do intervalo 0-1, no qual o valor $0(0 \%)$ significa uma completa falta de transparência de dados e o valor $1(100 \%)$ representa o mais alto nível de transparência. Para realizar a avaliação sobre a transparência de dados foram escolhidos todos os conjuntos publicados das cinco categorias citadas no início deste item.

$$
\mathrm{DT}=(\mathrm{A}+\mathrm{U}+(\mathrm{DR} / 3) / 3
$$

Para o GT foi utilizado o Corruptions Perception Indicator conforme indicação de 
Veljković, Bogdanović-Dinić e Stoimenov (2014). No caso do Brasil, o valor desse índice estava na posição 72 entre 177 países na última medição em 2013 (TRANSPARENCY INTERNATIONAL, 2013).

A Autenticidade (A) refere-se a fontes de dados (DS) e à Precisão e Integridade dos Dados (DAI). Veljković, Bogdanović-Dinić e Stoimenov (2014) atribuíram pesos diferentes para DS e para o DAI, conforme apresentado abaixo:

$$
\mathrm{A}=0,40 \times \mathrm{DS}+0,60 \times \mathrm{DAI}
$$

Segundo os autores, o governo deve publicar informações sobre fontes de dados em portais, tornando-o livremente disponível e acessível a todos os usuários. Além disso, o usuário deve ser capaz de fornecer uma classificação para cada fonte de dados, de acordo com a sua experiência na utilização dos dados, tendo a possibilidade de fornecer uma classificação aos outros usuários. Considerando esses aspectos, o modelo de referência utilizado propõe as seguintes características a serem avaliadas no processo de estimar um indicador de DS:

- Lista de fontes de dados disponíveis no portal de dados (F1) - 30\%;

- A possibilidade de conjuntos de dados publicados por uma revisão de dados específicos da fonte (F2) - 30\%;

- Disponível descrição da fonte de dados com informações básicas sobre os dados da fonte $(\mathrm{F} 3)-20 \%$;

- Informações sobre a classificação do usuário (F4) - $20 \%$.

A maior influência na construção do indicador DS é dada aos recursos marcados como F1 e F2, pois representam solicitações mais frequentes dos usuários. As características F3 e F4 também são importantes, mas são consideradas como tendo menos influência. Considerando todas as quatro características, as informações fornecidas pelo Governo (F1, F2 e F3) compõem $80 \%$ do valor do DS (conforme abaixo) enquanto que o feedback fornecido pelo usuário está envolvido com $20 \%$.

$$
\mathrm{DS}=0,30 \times \mathrm{F} 1+0,30 \times \mathrm{F} 2+0,20 \times \mathrm{F} 4+0,20 \times \mathrm{F} 4
$$

Os requisitos F1, F2 e F3 recebem o valor 1 caso sejam atendidos, caso contrário recebem 0 . O F4 representa um grau médio dimensionado entre 0 e 1 . O DS recebe valores entre 0 e 1 , sendo que 0 significa que nenhuma das características está presente em um portal de dados abertos.

O DS obtido pelo portal dados.rs.gov.br foi 0,8 nas cinco categorias que possuíam conjunto de dados disponibilizados. Os detalhes do levantamento são apresentados no 
Apêndice. $\mathrm{O}$ valor obtido pode ser considerado um bom resultado, indicando clareza e entendimento das fontes de dados que possuíam conjuntos de dados. Entretanto, faltou um aspecto importante, a possibilidade de avaliação (feedback) por parte dos usuários das fontes de dados, que afetará muito um outro indicador, o DAI, como será percebido a seguir.

Para fornecer um método de avaliação significativa e confiável ao DAI (Precisão e Integridade dos Dados), o modelo utilizado propõe a avaliação de três recursos:

- Classificação de governo (F5) - 35\%;

- Classificação do usuário (F6) - 35\%;

- Certificação de qualidade (F7) - 30\%

A classificação implica na existência de feedback fornecido pelo governo na precisão e integração para cada conjunto de dados publicados. No processo de avaliação, a nota média é definida entre os valores 0 e 1 . A classificação do usuário representa o feedback fornecido pelos usuários a respeito dos dados publicados. Para efeito de cálculo, deve ser levado em consideração o número de usuários que forneceram seus comentários.

O valor final de classificação do usuário é calculado como: usuários $x$ (avaliadores/números de downloads). As avaliações dos usuários representam a nota média escalada para o intervalo $(0,1)$, e os avaliadores representam o número de usuários que classificaram o conjunto de dados. A classificação fornecida pelo usuário é muito importante porque representa um ponto de vista, a opinião e a experiência do uso, por isso formam $70 \%$ do indicador DAI.

A terceira característica que compõem o DAI é a certificação de qualidade, que reconhece a existência de um documento de certificação para um conjunto de dados. A certificação de qualidade representa a prova eletrônica da precisão dos dados e integridade da informação contida. O valor final do recurso de DAI é calculado de acordo com a equação descrita abaixo:

$\mathrm{DAI}=0,35 \times \mathrm{F} 5+0,35 \times \mathrm{F} 6+0,30 \times \mathrm{F} 7$

Infelizmente, o portal dados.rs.gov.br, não possuía nenhum dos três recursos (F5, F6 e F7), por isso obteve 0 (zero) no indicador de Dados de Precisão e Integridade (DAI). O DAI tem seus valores dentro do intervalo entre 0 (zero) e 1 (um), o zero significa uma total falta de confiança na precisão e integridade dos dados, e 1 significa a satisfação máxima de todos os três recursos. O DAI com valor zero afetou negativamente o indicador de autenticidade (A) que obteve o valor 0,32 .

Segundo Veljković, Bogdanović-Dinić e Stoimenov (2014), a compreensibilidade de 
cada categoria de conjunto de dados e dos dados brutos precisa ser proporcionada aos usuários, algo que pode ser propiciado pela publicação de descrições textuais detalhadas de categorias de dados que expliquem os tipos de dados que são publicados em cada categoria, bem como descrições de cada conjunto de dados contido na categoria.

O indicador de compreensibilidade (U) permite verificar cada categoria de dados a partir de um conjunto de dados, verificando se há uma descrição para cada conjunto de dados dentro de cada categoria. O indicador de compreensibilidade é calculado com base nas características DCD e DSD, conforme a equação descrita abaixo. O indicador de compreensibilidade varia de 0 a 1 , o 1 indica a disponibilidade total de ambas as categorias de dados e as descrições do conjunto de dados. Por outro lado, se não existem descrições das categorias e conjuntos de dados, o indicador de compreensibilidade tem o valor 0 .

$$
\mathrm{U}=0,4 \times \mathrm{DCD}+0,6 \times \mathrm{DSD}
$$

O indicador Descrições de Categorias de Dados (DCD) é avaliado por três características:

- Descrição textual (34\%) (F8) - existência de uma descrição textual de dados para um conjunto de dados;

- Etiquetas (33\%) (F9) - existência de marcas pesquisáveis associadas a um conjunto de dados;

- Informações ligadas (33\%) (F10) - a ligação para a informação adicional em relação ao conjunto de dados.

Todos os três elementos são considerados como sendo igualmente importantes. A categoria é avaliada, com o valor de 1 - se existe a característica - ou 0 , se não existe. O DCD é calculado de acordo com a equação, demonstrada abaixo, resultando em valores no intervalo de 0 a 1, o 0 significa que nenhuma categoria tem descrição e o valor de 1 significa que todas as categorias têm descrições.

$$
\mathrm{DCD}=(0,34 \times \mathrm{F} 8+0,33 \times \mathrm{F} 9+0,33 \times \mathrm{F} 10)
$$

Os resultados do DCD foram os máximos possíveis (conforme demonstrado no Apêndice), pois todas as categorias possuíam as características avaliadas (F8, F9 e F10). Isso denota uma boa documentação das cinco categorias avaliadas e maior facilidade de localização da categoria dentro do portal.

A Descrição de Conjuntos de Dados é calculada com base nas mesmas três 
características do DCD, mas com relação a cada conjunto de dados, que é avaliado de 0 a 1 , seguindo a mesma equação da DCD. O resultado é o valor médio de todos os conjuntos de dados. Os valores DSD variam de 0 a 1 , onde 0 indica uma falta total de descrição dos dados, enquanto que 1 significa que todos os conjuntos de dados são adequadamente descritos. Os conjuntos de dados obtiveram a nota máxima nesse indicador, conforme mostrado no Apêndice, pois todos possuíam as características avaliadas (F8, F9 e F10). Isso destaca a boa documentação dos conjuntos de dados e a localização mais facilitada.

A Reutilização dos Dados (DR) é o último aspecto para determinar o grau de transparência dos Dados Abertos Governamentais no portal escolhido e refere-se ao fornecimento de dados em formatos abertos para que os usuários possam pesquisar, indexar e baixar os dados utilizando ferramentas comuns, sem qualquer conhecimento prévio das estruturas de dados. Veljković, Bogdanović-Dinić e Stoimenov (2014) utilizaram uma escala para calcular a reutilização de dados que consta em apêndice e que deve ser calculada pela avaliação do formato dos conjuntos de dados dentro de cada categoria de dado. $\mathrm{O}$ valor do indicador DR é um valor médio de reutilização dos conjuntos de dados, com valores de 0 a 3 , onde o valor 0 representa o menor nível de disponibilidade de dados e o valor 3 representa o mais alto nível de disponibilidade dos dados.

O resultado do indicador de Reutilização dos Dados (DR) consta no Apêndice, e indica que não está em nível ideal, mas em um nível satisfatório, obtendo um valor médio de 2,60 .

Por fim, pode-se avaliar o grau de transparência, conforme resultados mostrados no Apêndice. O grau de transparência, considerando a média das cinco categorias com conjunto de dados, no portal dados.rs.gov.br, foi de 0,57. O detalhamento sobre os valores obtidos em cada indicador constam no Apêndice.

\section{Considerações Finais}

A escolha do modelo de Veljković, Bogdanović-Dinić e Stoimenov (2014) mostrou-se acertada pela facilidade de entendimento dos critérios e a consequente facilidade de reaplicação futura, permitindo a comparação com demais portais nacionais e internacionais, servindo como um ótimo benchmarking. Como crítica remanesce apenas a necessidade de reavaliar o peso atribuído ao item Transparência do Governo (GT), que está vinculado a indicadores externos ao portal, como o Corruptions Perception Indicator, utilizado neste caso e indicado por Veljković, Bogdanović-Dinić e Stoimenov (2014) para valorar o GT. Esse indicador acaba influenciando demasiadamente o grau de Transparência do portal, mesmo 
quando a Transparência dos Dados (DT) é plena. Embora a transparência e o governo aberto contribuam para a redução dos níveis de corrupção, entende-se que esta medida não deveria estar vinculada à avaliação de dados de um portal de dados abertos uma vez que a redução dos níveis de corrupção envolve mais o uso de dados abertos pela sociedade (e a cobrança decorrente dos entes públicos) do que os dados disponibilizados. A medida da redução dos níveis de corrupção deveria ser, então, indireta.

O Grau de transparência do Portal dados.rs.gov.br obteve um bom índice, 56,8\%, que é considerado uma transparência moderada, conforme a classificação de Veljković, Bogdanović-Dinić e Stoimenov (2014), conforme consta no Apêndice. Outro dado importante, e que depende apenas dos responsáveis pelo portal, é a Transparência dos Dados, que obteve um indicador de 72,9\%, o que não é ruim, mas apresenta espaço para melhorias.

Em relação à quantidade de categorias presentes no portal dados.rs.gov.br, o Indicador Básico do Conjunto de Dados (BSD) obtido foi 0,56, o que indica que há muito a crescer, por meio da publicação e demais categorias consideradas primordiais na opinião de Veljković, Bogdanović-Dinić e Stoimenov (2014), como o Meio Ambiente, a Energia, a Infraestrutura e o Emprego, disponibilizadas diretamente no portal. Por outro lado, há categorias que foram priorizadas no contexto regional brasileiro, mas que não são valoradas pelo modelo utilizado. Isso instiga pesquisas futuras, que avaliem a valoração das categorias considerando os elementos de contexto, como, por exemplo, a legislação de cada país, pois a legislação indica em quais áreas o estado deve atuar e esta atuação deveria definir as categorias a serem pontuadas. Da mesma forma, a legislação indica dados que devem ser mantidos de forma confidencial.

Os resultados também indicam que ainda faltam várias funcionalidades, apontadas como importantes por Veljković, Bogdanović-Dinić e Stoimenov (2014). Entre as principais estão a falta de feedback por parte do usuário, a falta do uso de licenças em todas as publicações, a falta de atualização dos dados conforme a periodicidade proposta pelo portal, a falta da definição de periodicidade da atualização dos dados e a falta de classificação por todos os gêneros: governo; cidadão e qualidade. Como discutido anteriormente, também há muitas categorias que têm baixa pontuação em relação à abertura dos dados, e há também muitos indicadores que apontam para potenciais fraquezas. A análise da transparência de dados demonstrou deficiências significativas sobre os indicadores, em especial a Autenticidade dos Dados (A), a Reusabilidade dos Dados (DR) e a Transparência dos Dados (DT).

Os resultados da análise do Portal dados.rs.gov.br podem ser considerados bons para o 
momento. No entanto, indicam aspectos que podem ser aprimorados pelos gestores públicos, e propiciam um comparativo entre um contexto regional brasileiro e o americano (em relação ao DAG), suscitando a evolução de novos modelos de mensuração que melhor se adequem as necessidades de cada contexto, por intermédio de novas pesquisas. As contribuições deste artigo vão além da indicação de aspectos em que este portal pode melhorar, já que as análises feitas e os aspectos considerados podem ser úteis para outros portais melhorarem o nível de transparência. Igualmente, portais ainda em elaboração também podem verificar um possível caminho a seguir. Outra contribuição é a verificação da aplicabilidade do framework desenvolvido por Veljković, Bogdanović-Dinić e Stoimenov (2014) no contexto nacional. 


\section{REFERÊNCIAS}

BRASIL. LEI No 12.527, DE 18 DE NOVEMBRO DE 2011. Regula o acesso a informações previsto no inciso XXXIII do art. 5o, no inciso II do § 30 do art. 37 e no $\S 20$ do art. 216 da Constituição Federal; altera a Lei no 8.112, de 11 de dezembro de 1990; revoga a Lei no 11.111, de 5 de maio de 2005, e dispositivos da Lei no 8.159, de 8 de janeiro de 1991; e dá outras providências.

CONTROLADORIA-GERAL DA UNIÃO. Manual da Lei de Acesso à Informação para

Estados e Municípios. . Brasília: [s.n.], 2013. Disponível em:

$<$ http://www.cgu.gov.br/publicacoes/BrasilTransparente/Manual_LAI_EstadosMunicipios.pd $\mathrm{f}>$.

DA SILVA JÚNIOR, A. O perfil ideal do contador público frente à responsabilidade social. Revista Economia \& Gestão, v. 1, n. 2, 2008.

DAVIES, T. Open Data Barometer 2013 Global Report. World Wide Web Foundation and Open Data Instituto. 2013. Disponível em: http://www.opendataresearch.org/dl/odb2013/Open-Data-Barometer-2013-Global-

Report.pdf. Acesso 14 de jun. de 2014.

DAWES S.S. Stewardship and usefulness: Policy principles for information-based transparency. Government Information Quarterly, v. 27, p. 377-383, 2010.

DAWES S. S; E HELBIG N. Information Strategies for Open Government: Challenges and Prospects for Deriving Public Value from Government Transparency. In: Electronic Government: Lecture Notes in Computer Science, M.A. Wimmer et al. (Eds.): EGOV 2010, LNCS 6228, p. 50-60, 2010. Disponível em: http://www.ctg.albany.edu/publications/journals/ifip_2010_opengov/ifip_2010_opengov.pdf. Acesso em 29 jun. 2014.

DE ANDRADE, S. A.; TEIXEIRA, A.; FORTUNATO, G. Influência dos Gastos Públicos Sociais Sobre o PIB dos Municípios do Estado de Minas Gerais. DOI-10.5752/P. 19846606.2014 v14n35p112. Revista Economia \& Gestão, v. 14, n. 35, p. 112-130, 2014.

DE FERRANTI, D.M.; JACINTO, J.; ODY, A. J.; RAMSHAW, G., How to Improve Governance: a New Framework for Analysis and Action. Washington, Brookings Institution Press, 2009.

DELOITTE ANALYTICS, Open growth. Stimulating demand for open data in the UK. 2013. Disponível em: www.deloitte.com/view/en_gb/uk/market-insights/deloitteanalytics/bfb570a79416b310VgnVCM1000003256f70aRCRD.htm. Acesso 14 de jun. de 2014.

DNIT. Departamento Nacional Departamento Nacional de Infraestrutura de Transportes. Estatísticas de Acidentes de Trânsito. 2014. Disponível em: http://www.dnit.gov.br/rodovias/operacoes-rodoviarias/estatisticas-de-acidentes. Acesso em 21 de jun. de 2014.

FLICK, U. Introdução à pesquisa qualitativa. 3. ed. Porto Alegre: Artmed, 2009.

HARRISON, T. M. et al. Open government and e-government: Democratic challenges from a public value perspective, Information Polity, v. 17, p. 83-97, 2012. 
JANSSEN, M.; ZUIDERWIJK, A. Infomediary Business Models for Connecting Open Data Providers and Users. Social Science Computer Review, n.32, issue 5, 2014.

KOSKI, H. Does marginal cost pricing of public sector information spur firm growth? ETLA Discussion Papers, The Research Institute of the Finnish Economy (ETLA), 2011.

LEE, G.; KWAK, Y. H. Open government implementation model: A stage model for achieving increased public engagement. Paper presented at the 12th Annual International Digital Government Research Conference: Digital Government Innovation in Challenging Times, pp. 254-261, 2011.

MAGALHAES, G.; ROSEIRA, C.; STROVER, S. Open government data intermediaries: a terminology framework. In: Proceedings of the 7th International Conference on Theory and Practice of Electronic Governance. ACM, 2013. p. 330-333.

MCDERMOTT, P. Building open government. Government Information Quarterly, v. 27, n. 4, p. 401-413, 2010.

MICHENER, Robert Gregory; MONCAU, Luiz Fernando Marrey; VELASCO, Rafael. Estado Brasileiro e Transparência: avaliando a aplicação da Lei de Acesso à Informação. Avaliação nacional de Transparência Governamental: usos e desafios da Lei de Acesso à Informação - Lei 12.527. Rio de Janeiro: [s.n.], 2014.

OECD - Manual de Oslo - Diretrizes para a coleta e interpretação de dados sobre Inovação. 3a ed., Tradução FINEP, 2007, Disponível em: www.finep.org.br. Acesso em 21 de jun. de 2014.

OGP - OPEN GOVERNMENT PARTNERSHIP. About. 2014. Disponível em: www.opengovpartnership.org/about. Acesso em 23 jun. 2014.

ONTSI - Observatorio Nacional de las Telecomunicaciones y de la Sociedad de la Información. Annual Report. Characterization Study of the Infomediary Sector, July 2012. 2012. Disponível

em: http://datos.gob.es/sites/default/files/121001\%20RED\%20007\%20Final\%20Report_2012\%20 Edition_\%20vF_en.pdf. Acesso em: 15 de jun. de 2014.

OPEN GOVERNMENT DATA. Eight principles of open government data. Disponível em: http://resource.org/8_principles.html. Acesso em: 22 de abr. de 2015.

SCHOLL, H. J. Five trends that matter: Challenges to 21 st century electronic government. Information Polity, v. 17, p. 317-327, 2012.

TRANSPARENCY INTERNATIONAL. Corruption Perceptions Index. 2013. Disponível em: http://www.transparency.org/cpi2013. Acesso em 16 jun. 2014.

UBALDI, B. Open Government Data: Towards Empirical Analysis of Open Government Data Initiatives. OECD Working Papers on Public Governance, No. 22, OECD Publishing, 2013.

VELJKOVIĆ, N.; BOGDANOVIĆ-DINIĆ, S.; STOIMENOV, L. Benchmarking open government: An open data perspective. Government Information Quarterly, v. 31, n. 2, p. 278-290, 2014.

VICKERY, Graham. Review of recent studies on PSI re-use and related market developments. Information Economics, Paris, 2011.

YILDIZ, M.; E-government research: Reviewing the literature, limitations, and ways forward. Government Information Quarterly, v. 24, n.3, p .646-665, 2007. 
ZUIDERWIJK, A.; JANSSEN, M. Open data policies, their implementation and impact: A framework for comparison. Government Information Quarterly, v. 31, n. 1, p. 17-29, 2014. 


\section{APÊNDICE}

Indicador de abertura de dados (DO) para o portal dados.rs.gov.br

\begin{tabular}{|c|c|c|c|c|c|c|c|c|c|c|c|}
\hline 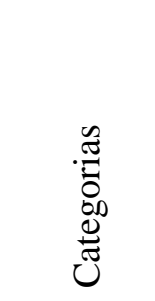 & 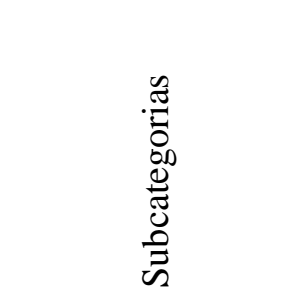 & 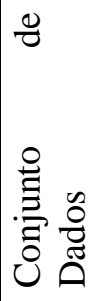 & 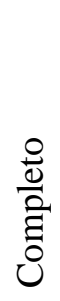 & 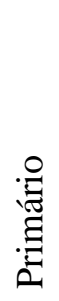 & $\frac{\stackrel{n}{\Xi}}{\stackrel{\Xi}{Z}}$ & 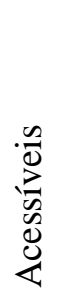 & 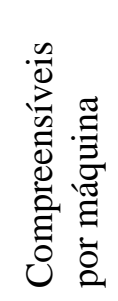 & 章 & 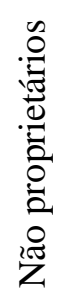 & 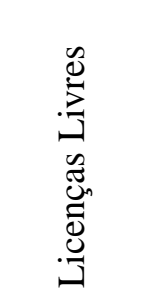 & 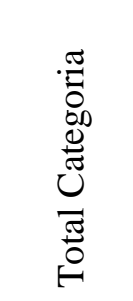 \\
\hline \multirow{2}{*}{ Finanças } & Finanças & 8 & 1 & 1 & $0,4125^{*}$ & 1 & $\begin{array}{c}0,8125 \\
*\end{array}$ & 1 & 1 & $0,3750^{*}$ & 0,825 \\
\hline & Desenvolvimento & 4 & 1 & 1 & 0,3 & 1 & 1 & 1 & 1 & 0 & 0,7875 \\
\hline \multicolumn{2}{|l|}{ Saúde } & 5 & 1 & 1 & 0,3 & 1 & 1 & 1 & 1 & 0 & 0,7875 \\
\hline \multicolumn{2}{|l|}{ Educação } & 17 & 1 & 1 & 0,3 & 1 & 0,5 & 1 & 1 & 0 & 0,73 \\
\hline \multicolumn{2}{|c|}{ Transporte (Trânsito) } & 7 & 1 & 1 & $0,3857^{*}$ & 1 & $0,5^{*}$ & 1 & 1 & $0,2857^{*}$ & 0,7714 \\
\hline \multicolumn{2}{|c|}{ População } & 4 & 1 & 1 & 0,3 & 0 & 1 & 1 & 1 & 0 & 0,6625 \\
\hline \multicolumn{2}{|c|}{ TOTAL (DO) } & & & & & & & & & & 36,4714 \\
\hline
\end{tabular}

Valores médios obtidos conforme Quadro 1.

*Alguns conjuntos de dados não obtiveram o mesmo valor, influenciando na média da categoria.

Resultado da avaliação das fontes de dados abertos (DS)

\begin{tabular}{|c|c|c|c|c|c|c|c|}
\hline 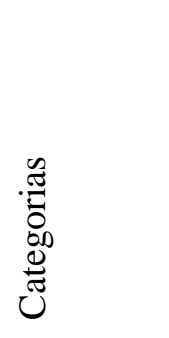 & 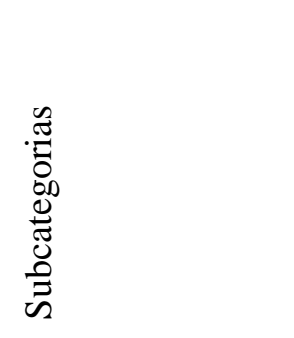 & 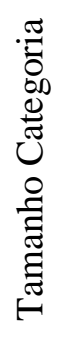 & 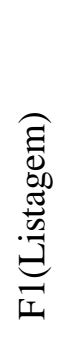 & 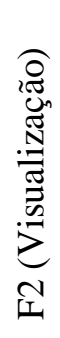 & 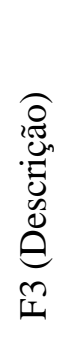 & 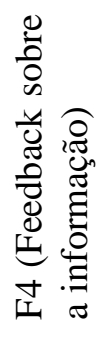 & 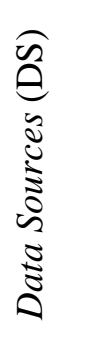 \\
\hline \multirow[t]{2}{*}{ Finanças } & Finanças & 8 & 1 & 1 & 1 & 0 & 0,8 \\
\hline & Desenvolvimento & 4 & 1 & 1 & 1 & 0 & 0,8 \\
\hline \multicolumn{2}{|l|}{ Saúde } & 5 & 1 & 1 & 1 & 0 & 0,8 \\
\hline \multicolumn{2}{|l|}{ Educação } & 17 & 1 & 1 & 1 & 0 & $\overline{0,8}$ \\
\hline \multicolumn{2}{|c|}{ Transporte (Trânsito) } & 7 & 1 & 1 & 1 & 0 & 0,8 \\
\hline \multicolumn{2}{|l|}{ População } & 4 & 1 & 1 & 1 & 0 & 0,8 \\
\hline \multicolumn{2}{|c|}{ Média Final DS } & & & & & & 0,8 \\
\hline
\end{tabular}

Fórmula para cálculo do DS:

$$
\mathrm{DS}=0,30 \times \mathrm{F} 1+0,30 \times \mathrm{F} 2+0,20 \times \mathrm{F} 4+0,20 \times \mathrm{F} 4
$$

Os requisitos F1, F2 e F3 recebem o valor 1 caso sejam atendidos, caso contrário recebem 0 . O F4 representa um grau médio dimensionado entre 0 e 1 . O DS recebe valores entre 0 e 1 , sendo que 0 significa que nenhuma das características está presente em um portal de dados abertos. 
Resultado da avaliação das Descrições de Categorias de Dados (DCD)

\begin{tabular}{|l|l|c|c|c|c|}
\hline Categorias & Subcategorias & $\begin{array}{c}\text { Tamanho } \\
\text { Categoria }\end{array}$ & $\begin{array}{c}\text { F8 - DCD } \\
\text { (Descrição textual) }\end{array}$ & $\begin{array}{c}\text { F9 - DCD } \\
\text { (Tags) }\end{array}$ & $\begin{array}{c}\text { F10 - DCD } \\
\text { (Link para } \\
\text { informações } \\
\text { adicionais) }\end{array}$ \\
\hline \multirow{2}{*}{ Finanças } & Finanças & 8 & 1 & 1 & 1 \\
\cline { 2 - 6 } & Desenvolvimento & 4 & 1 & 1 & 1 \\
\hline Saúde & 5 & 1 & 1 & 1 \\
\hline Educação & 17 & 1 & 1 & 1 \\
\hline \multicolumn{2}{|l|}{ Transporte (Trânsito) } & 7 & 1 & 1 & 1 \\
\hline \multicolumn{2}{l|}{ População } & 4 & 1 & 1 & 1 \\
\hline
\end{tabular}

Valor de 1 se existe a característica ou 0, se não existe. O DCD é calculado de acordo com a equação, demonstrada abaixo, resultando em valores no intervalo de 0 a 1 , o 0 significa que nenhuma categoria tem descrição e o valor de 1 significa que todas as categorias têm descrições.

$\mathrm{DCD}=(0,34 \times \mathrm{F} 8+0,33 \times \mathrm{F} 9+0,33 \times \mathrm{F} 10)$

Resultado da avaliação das fontes de dados abertos (DSD)

\begin{tabular}{|l|l|c|c|c|c|c|}
\hline Categorias & Subcategorias & $\begin{array}{c}\text { Tamanho } \\
\text { Categoria }\end{array}$ & $\begin{array}{c}\text { F8 - DSD } \\
\text { (Descrição } \\
\text { textual) }\end{array}$ & $\begin{array}{c}\text { F9 - } \\
\text { DSD } \\
\text { (Tags) }\end{array}$ & $\begin{array}{c}\text { F10 - DSD } \\
\text { (Link para } \\
\text { informações } \\
\text { adicionais) }\end{array}$ & $\begin{array}{c}\text { Descrição } \\
\text { dos Cj. de } \\
\text { Dados } \\
\text { (DSD) }\end{array}$ \\
\hline \multirow{2}{*}{ Finanças } & Finanças & 8 & 1 & 1 & 1 & 1 \\
\cline { 2 - 7 } & Desenvolvimento & 4 & 1 & 1 & 1 & 1 \\
\hline Saúde & 5 & 1 & 1 & 1 & 1 \\
\hline \multicolumn{2}{|l|}{ Educação } & 17 & 1 & 1 & 1 & 1 \\
\hline \multicolumn{2}{|l|}{ Transporte (Trânsito) } & 7 & 1 & 1 & 1 & 1 \\
\hline \multicolumn{2}{l|l}{ População } & 4 & 1 & 1 & 1 & 1 \\
\hline
\end{tabular}

Os valores DSD variam de 0 a 1 , onde 0 indica uma falta total de descrição dos dados, enquanto que 1 significa que todos os conjuntos de dados são adequadamente descritos.

Pontuação para a reutilização dos dados.

\begin{tabular}{|c|l|}
\hline Valor & Situação \\
\hline 0 & Sem dados \\
\hline 1 & Arquivos em formato PDF ou XLS \\
\hline 2 & $\begin{array}{l}\text { Arquivos e formato legível por máquina. Formato CSV. Estruturado em formato } \\
\text { não proprietário }\end{array}$ \\
\hline 3 & $\begin{array}{l}\text { Arquivos em formato XLM ou RDF (Resource Description Framework) } \\
\text { (Notação semântica) }\end{array}$ \\
\hline
\end{tabular}

Fonte: Veljković, Bogdanović-Dinić e Stoimenov (2014) 
Níveis de reutilização dos dados.

\begin{tabular}{|l|l|c|c|}
\hline Categorias & Subcategorias & Tamanho da Categoria & DR (reusabilidade) \\
\hline \multirow{2}{*}{ Finanças } & Finanças & 8 & $2,625^{*}$ \\
\cline { 2 - 4 } & Desenvolvimento & 4 & 3 \\
\hline Saúde & 5 & 3 \\
\hline Educação & 17 & 2 \\
\hline Transporte (Trânsito) & 7 & 2 \\
\hline População & 4 & 2,60 \\
\hline Média do DR & & 3 \\
\hline
\end{tabular}

O valor do indicador DR é um valor médio de reutilização dos conjuntos de dados, com valores de 0 a 3 , onde o valor 0 representa o menor nível de disponibilidade de dados e o valor 3 representa o mais alto nível de disponibilidade dos dados.

* Alguns conjuntos de dados não obtiveram o mesmo valor, influenciando na média da categoria.

A Autenticidade (A) refere-se a fontes de dados (DS) e à Precisão e Integridade dos Dados (DAI).

$$
\mathrm{DAI}=0,35 \times \mathrm{F} 5+0,35 \times \mathrm{F} 6+0,30 \times \mathrm{F} 7
$$

F5 - Classificação (feedback) de governo

F6 - Classificação (feedback) do usuário

F7 - Certificação (feedback) de qualidade

A classificação implica na existência de feedback fornecido pelo governo na precisão e integração para cada conjunto de dados publicados. No processo de avaliação, a nota média é definida entre os valores 0 e 1 . A classificação do usuário representa o feedback, fornecido pelos usuários, a respeito dos dados publicados. Para efeito de cálculo, deve ser levado em consideração o número de usuários que forneceram seus comentários.

$$
\mathrm{A}=0,40 \times \mathrm{DS}+0,60 \times \mathrm{DAI}
$$

O indicador de compreensibilidade (U) permite verificar cada categoria de dados a partir de um conjunto de dados, verificando se há uma descrição para cada conjunto de dados dentro de cada categoria. O indicador de compreensibilidade é calculado com base nas características DCD e DSD, conforme a equação descrita abaixo:

$$
\mathrm{U}=0,4 \times \mathrm{DCD}+0,6 \times \mathrm{DSD}
$$

O indicador de Transparência dos Dados (DT) é calculado como uma média dos valores de autenticidade (A), Compreensibilidade (U) e reutilização dos dados, conforme apresentado abaixo. Este indicador tem valores a partir do intervalo $(0,1)$, onde o valor $0(0 \%)$ significa uma completa falta de transparência de dados e o valor 1 $(100 \%)$ representa o mais alto nível de transparência. Para realizar a avaliação sobre a transparência de dados foram escolhidos todos os conjuntos publicados das cinco categorias citadas no início deste item.

$$
\mathrm{DT}=(\mathrm{A}+\mathrm{U}+(\mathrm{DR} / 3) / 3
$$

Para o GT foi utilizado o Corruptions Perception Indicator conforme indicação de Veljković, Bogdanović-Dinić e Stoimenov (2014). No caso do Brasil, o valor desse índice estava na posição 72 entre 177 países na última medição em 2013 (TRANSPARENCY INTERNATIONAL, 2013). 
Valores para cálculo de GT - Grau de Transparência

\begin{tabular}{|c|c|c|c|c|c|c|c|c|}
\hline 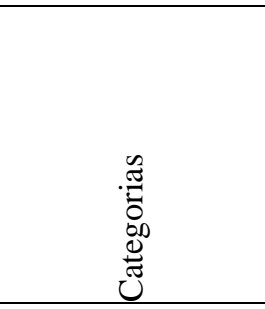 & 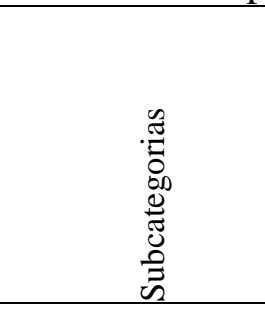 & 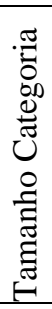 & 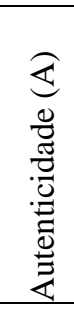 & 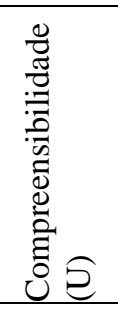 & 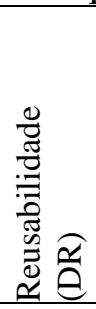 & 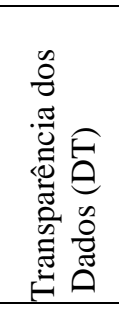 & 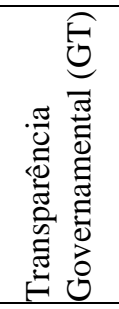 & 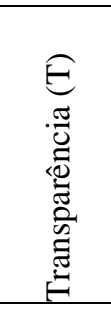 \\
\hline \multirow{2}{*}{ Finanças } & Finanças & 8 & 0,32 & 1,0000 & 2,625 & 0,7317 & 0,4066 & 0,5691 \\
\hline & Desenvolvimento & 4 & 0,32 & 1,0000 & 3 & 0,7733 & 0,4066 & 0,5900 \\
\hline Saúde & & 5 & 0,32 & 1,0000 & 3 & 0,7733 & 0,4066 & 0,5900 \\
\hline Educação & & 17 & 0,32 & 1,0000 & 2 & 0,6622 & 0,4066 & 0,5344 \\
\hline $\begin{array}{l}\text { Transporte } \\
\text { (Trânsito) }\end{array}$ & & 7 & 0,32 & 1,0000 & 2 & 0,6622 & 0,4066 & 0,5344 \\
\hline População & & 4 & 0,32 & 1,0000 & 3 & 0,7733 & 0,4066 & 0,5900 \\
\hline $\begin{array}{l}\text { Grau de } \\
\text { Transparência }\end{array}$ & & & & & & 0,7294 & & 0,5680 \\
\hline
\end{tabular}

$\mathrm{O}$ indicador $\mathrm{T}$ tem seus valores a partir do intervalo $(0,1)$, onde 0 (zero) significa a falta de transparência e 1 (um) está associada à alta transparência.

Níveis de Transparência para um portal de Dados Abertos Governamentais

\begin{tabular}{|c|c|c|c|l|}
\hline $\begin{array}{l}\text { Autenticidade } \\
(\mathrm{A})\end{array}$ & $\begin{array}{l}\text { Compreensibilidade } \\
(\mathrm{U})\end{array}$ & $\begin{array}{l}\text { Reusabilidade } \\
(\mathrm{DR})\end{array}$ & $\begin{array}{l}\text { Transparência } \\
\text { dos Dados (DT) }\end{array}$ & $\begin{array}{l}\text { Nível de } \\
\text { Transparência }\end{array}$ \\
\hline $0-0,05$ & $0-0,05$ & $0-0,05$ & $0-5 \%$ & $\begin{array}{l}\text { Inicial } \\
\text { (cradle) }\end{array}$ \\
\hline $0,06-0,2$ & $0,06-0,2$ & $0,06-0,2$ & $6 \%-20 \%$ & $\begin{array}{l}\text { Transparência } \\
\text { Básica }\end{array}$ \\
\hline $0,21-0,75$ & $0,21-0,75$ & $0,21-0,75$ & $21 \%-75 \%$ & $\begin{array}{l}\text { Transparência } \\
\text { moderada }\end{array}$ \\
\hline $0,76-0,9$ & $0,76-0,9$ & $0,76-0,9$ & $76 \%-90 \%$ & Transparente \\
\hline Maior que 0,9 & Maior que 0,9 & Maior que 0,9 & Maior que 0,9\% & $\begin{array}{l}\text { Alta } \\
\text { Transparência }\end{array}$ \\
\hline
\end{tabular}

Fonte: Veljković, Bogdanović-Dinić e Stoimenov (2014) 